\title{
Transmission Span Optimization in Fiber Systems With Cavity and Random Distributed Feedback Ultralong Raman Laser Amplification
}

\author{
Giuseppe Rizzelli, Paweł Rosa, Pedro Corredera, and Juan Diego Ania-Castañón
}

\begin{abstract}
Optimal design of a Raman-amplified periodic cell depends strongly on the constraints set by overall system requirements. The best possible tradeoff in terms of cost-effectiveness, nonlinearity, pumping efficiency, optical signal-to-noise ratio (OSNR), and tolerance to relative intensity noise transfer varies depending on characteristics, such as total transmission length, desirable number of spans or power budget requirements. Here, we perform a thorough experimental and theoretical study of the best performing configurations for second-order amplification over a broad range of applications, with span lengths ranging from 10 to $150 \mathrm{~km}$. Results highlight the presence of different regimes in which different contributions to noise become dominant. We find that ultralong Raman fiber laser cells with high forward pumping are to be preferred in unrepeatered transmission applications, whereas in long-haul transmission a shorter cell based on random distributed feedback lasing can be a better option as it enables low relative intensity noise transfer with a higher output OSNR at the cost of a reduced pumping efficiency.
\end{abstract}

Index Terms-Fiber optics, optical communications, Raman amplifier, relative intensity noise, ultra-long Raman fiber laser amplifier.

\section{INTRODUCTION}

$\mathbf{R}$ AMAN amplification has become a key enabling technology in different applications for telecommunications thanks to its flexibility and typically excellent noise performance. When compared to traditional EDFA-based systems Raman amplifiers can enable data transmission over longer distances and a wider bandwidth relying on the currently installed

Manuscript received July 28, 2017; revised October 23, 2017; accepted October 23, 2017. Date of publication October 25, 2017; date of current version November 16, 2017. This work was supported in part by the People Programme of the European Union's Seventh Framework Programme FP7 under Grant 608099 and Programme H2020 under Grant 748767, in part by the Spanish MINECO under Grant TEC2015-71127-C2, and in part by the Comunidad de Madrid under Grant S2013/MIT-2790-SINFOTON-CM. (Corresponding author: Giuseppe Rizzelli.)

G. Rizzelli, P. Corredera, and J. D. Ania-Castañón are with the Instituto de Óptica "Daza de Valdés," CSICMadrid 28006, Spain (e-mail: giuseppe. rizzelli@csic.es; p.g.rosa@icloud.com; juan.diego@io.cfmac.csic.es).

P. Rosa was with the Instituto de Óptica "Daza de Valdés," CSICMadrid 28006, Spain. He is now with the National Institute of TelecommunicationsWarsaw 04894, Poland (e-mail: p.corredera@csic.es).

Color versions of one or more of the figures in this paper are available online at http://ieeexplore.ieee.org.

Digital Object Identifier 10.1109/JLT.2017.2766685 fiber infrastructure. Thus, over the past few years, various ultralong Raman laser-based amplifier configurations have been proposed [1]-[3] which have unlocked record performances in a variety of applications. Discrete broadband Raman amplification [4] and distributed amplification, both unrepeatered and long-haul [5]-[10], provide tangible improvements but are usually limited by two main drawbacks: low pumping efficiency and Relative Intensity Noise (RIN) transfer from the pumps to the signal [11]-[14]. For a given number of channels and transmission format, the required pump power mainly depends on the medium properties and, therefore there is little room for improvement if standard single mode fibers are to be used. Conversely, there exist a number of ways to mitigate RIN through system design while preserving the improved balance between ASE noise and nonlinearity typically provided by Raman amplifiers. For some low-gain applications, semiconductor pump lasers can be used that are inherently less noisy than fiber lasers, but these can only supply powers up to a few hundreds of $\mathrm{mW}$. In more power-consuming applications, fiber pump lasers are usually deployed in conjunction with different cavity setups, pumping schemes and nonlinearity compensation techniques [15]-[17]: it has been recently demonstrated that a 2nd-order random Distributed Feedback (rDFB) architecture with a halfopen cavity [3] can remarkably tolerate bidirectional pumping paying a strongly reduced price in terms of Q penalty due to RIN transfer [18] from the noisy fiber pump lasers. Likewise, we have shown that in 2nd-order amplification the maximum admissible forward (FW) pump contribution before RIN build-up depends on the amount of backreflected light at the front-end of the cavity [19]. To the best of our knowledge none of the previous works has dealt with an in-depth study of cavity operation, but rather with the transmission performance of a specific scheme. Here we present a thorough optimization of cavity parameters such as length, FW pump ratio (FPR) and front-end FBG reflectivity for 2nd-order ultralong Raman fiber laser amplifiers, aiming to improve transmission performance. The optimization is carried out through careful characterization of the input RIN of the laser pumps, the signal output RIN, the optical signal-tonoise ratio (OSNR) and the pump power requirements for full signal power recovery after the transmission span. We believe this study can facilitate the system design process by helping in addressing critical performance issues common to a broad range of operational conditions. 


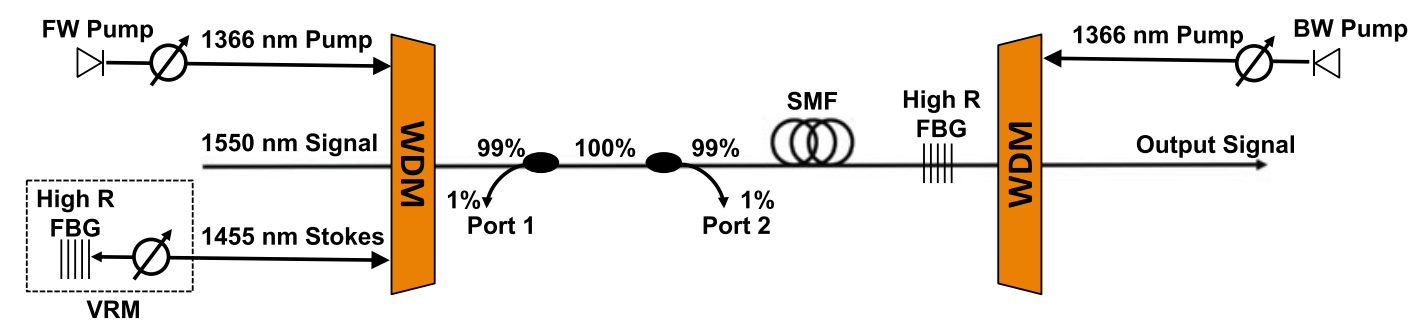

Fig. 1. Experimental setup of a 2nd-order Raman amplifier with variable front-end reflectivity.

\section{EXPERIMENTAL LAYOUT}

The bench-top implementation of the 2nd-order URFL under investigation was first proposed in [19] and is shown in Fig. 1. Two depolarized pump lasers provide 2nd-order pumping at $1366 \mathrm{~nm}$. At each pump output a variable optical attenuator (VOA) ensures that only the pump power changes, whereas the pump RIN is fixed. Two wavelength division multiplexers (WDMs) couple the pumps and the continuous wave signal at $1550 \mathrm{~nm}$ into the cavity. The cavity is delimited by two high reflectivity fiber Bragg gratings (FBG) at each side of a standard SMF span. The gratings have a $1 \mathrm{~nm}$ bandwidth centered around $1455 \mathrm{~nm}$ to assist the generation of the first Stokes component. The input side FBG is placed at the $1455 \mathrm{~nm}$ port of the WDM and combined with a VOA to form a variable reflectivity module (VRM) that can provide any reflectivity from $0 \%$ to $30 \%$. The $0 \%$ front-end reflectivity case (no FBG, angled fiber termination) corresponds to a half-open rDFB configuration, in which feedback is provided not by the grating, but distributedly through Rayleigh backscattering [3], [7], [8]. Lastly, two 99/1 couplers located inside the cavity allow for real time monitoring of the incident and backreflected light at the input side.

Signal input power is fixed at $-10 \mathrm{dBm}$ to minimize the impact of pump depletion or nonlinear effects in our analysis of the relative contribution of the different noise sources, but is nevertheless a realistic input power value for a broad range of applications, as the optimal launch power has been shown to be in between $-10 \mathrm{dBm}$ and $-4 \mathrm{dBm}$ in most of the experimental setups studied to date with this kind of amplification schemes [6]-[8]. Since the amount of gain provided by forward pump power is key to the performance of a Raman amplifier [7]-[11], as it is responsible for most of the RIN transfer to the signal, our analysis aims at showing the evolution of system parameters as a function of the ratio of the FW pump power to the total pump power, while the latter is adjusted to fully compensate for span losses at the amplifier output. We refer to this variable as the forward pump ratio (FPR).

The primary (second-order) pumps are two high-RIN fiber lasers capable of emitting up to $8 \mathrm{~W} \mathrm{CW}$ pump power. Their output RIN as a function of the output power is shown in Fig. 2. Fig. 2(a) displays the FW pump RIN measured with a $125 \mathrm{MHz}$ bandwidth low-noise photodetector in the $[9 \mathrm{kHz}$, $1 \mathrm{MHz}$ ] RF frequency range which, simulations show, is where the RIN transfer function $3 \mathrm{~dB}$ corner frequency occurs. Fig. 2(b) presents the pumps output RIN dependence on the output power by depicting the integral of the curves in Fig. 2(a) for both FW and backward (BW) pump. Both lasers seem to display

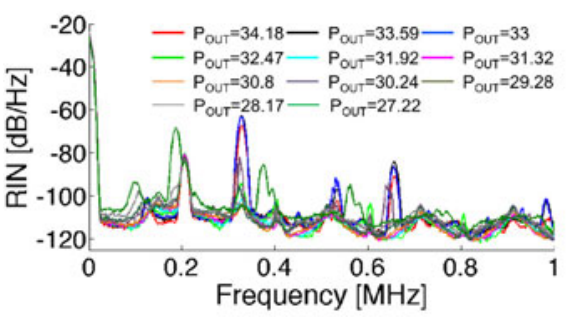

(a)

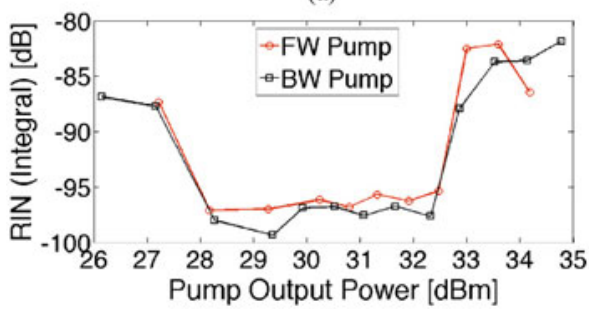

(b)

Fig. 2. FW pump output RIN (a) and pumps output RIN integral over the $[9 \mathrm{kHz}, 1 \mathrm{MHz}]$ frequency interval for different output power values.

minimum integrated output RIN in between $28 \mathrm{dBm}$ and $32.5 \mathrm{dBm}$. Unfortunately for the purpose of this study we needed each of the pumps to be able to single-handedly pump the cavity in order to span all of the possible FPRs while keeping the pumps RIN fixed, thus we set the FW and BW pump powers to $34.2 \mathrm{dBm}$ and $34.8 \mathrm{dBm}$ respectively.

\section{EXPERIMENTAL RESULTS}

Several cavity designs have been evaluated in relation to their key performance indicators. Total pump power needed to achieve zero net gain, OSNR over a $0.1 \mathrm{~nm}$ bandwidth, and output signal RIN have been measured as a function of the FPR for various cavity lengths and front-end reflectivities. Reflectivity was calculated as the ratio between the optical powers at Port 2 and Port 1 measured through an optical signal analyzer at the FBG central wavelength. The amplifier length ranges from very short $(10 \mathrm{~km})$ to quite long $(150 \mathrm{~km})$. Although short lengths are commonly used in practice for discrete amplification only, and due to their generally lower power requirements might in some applications rely on low-RIN semiconductor laser pumps instead of noisier fiber pump lasers, they have been included in our analysis in order to have a full picture of the evolution of system response. Also, short length URFL schemes have been demonstrated to provide simultaneous spectral and spatial trans- 


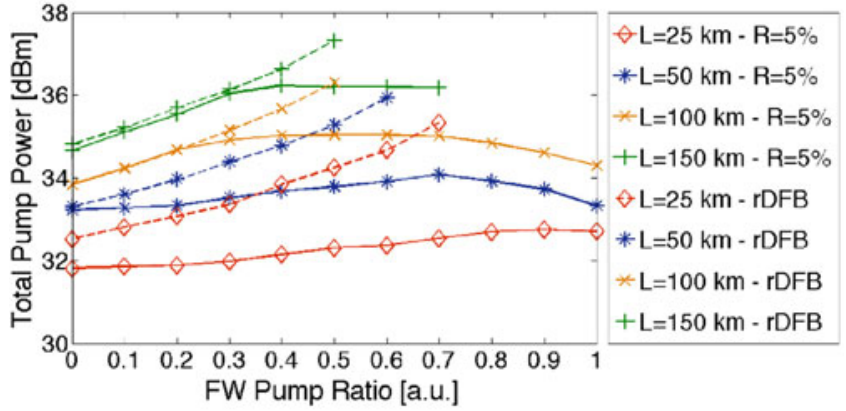

(a)

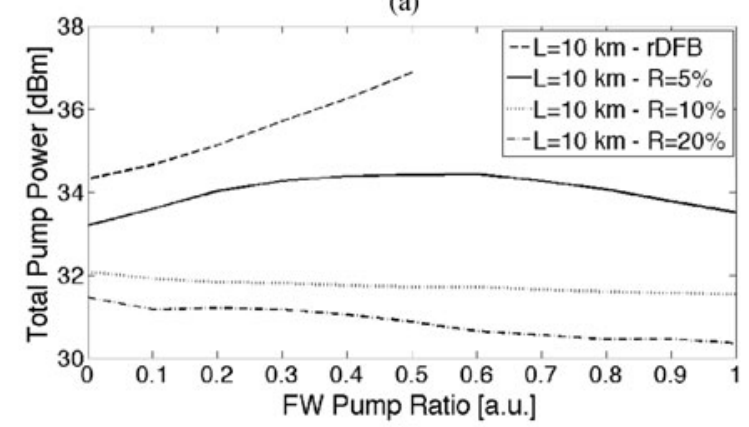

(b)

Fig. 3. Total pump power required to achieve zero net gain as a function of the FPR for (a) fourdifferent cavity lengths in the rDFB and 5\% reflectivity cases and (b) for a $10 \mathrm{~km}$ cavity and front reflectivity ranging from $0 \%$ to $20 \%$.

parency [20], and thus have interest on their own as a potential framework for the study of nonlinear transmission systems.

Fig. 3 shows how the sum of FW and BW pump powers evolves due to an increase of the VRM reflectivity as we gradually change the pumping scheme from purely counterpropagating $(\mathrm{FPR}=0)$ to co-propagating $(\mathrm{FPR}=1)$. The drop in pumping efficiency due to the absence of a reflector that helps the creation of the $1455 \mathrm{~nm}$ lasing is very clear for short cells up to $50 \mathrm{~km}$ (Fig. 3(a)): a reflectivity as low as $5 \%$ at the input side is sufficient to lower the amount of required pump power by $2 \mathrm{~dB}$ and $1.5 \mathrm{~dB}$ for a $25 \mathrm{~km}$ and $50 \mathrm{~km}$ cell respectively, when compared to the rDFB configuration at a $50 \%$ FPR. In the longer cells the pumps experience a higher attenuation, hence the backward (BW) pump power reaching the input side is too low to have any meaningful effect on FW Stokes generation and the $5 \%$ reflectivity cavity behaves similarly to the rDFB in configurations with low FW pumping. However, as the FW pump contribution grows, the FW $1455 \mathrm{~nm}$ Stokes is more efficiently generated thanks to the feedback provided by the front FBG and the overall pumping efficiency improves.

The special case of an extremely short $10 \mathrm{~km}$ cavity is depicted as an example in Fig. 3(b). The SMF length in this case is too short to be able to generate enough Raman gain inside the cavity and it results in a higher required pump power, comparable to the power necessary to pump $150 \mathrm{~km}$ in a rDFB design. The power efficiency improvement yielded by a higher front reflectivity is remarkable: about $6 \mathrm{~dB}$ can be saved at a $50 \%$ FPR using a 20\% FBG, nevertheless such a short cavity is still less efficient than the $25 \mathrm{~km}$ one and, in some cases, than the $50 \mathrm{~km}$ one.
Pumping efficiency is a crucial design parameter due to the energy consumption and the safety concerns it brings about in certain scenarios. If we assume that these aspects of the system design are addressed properly, Raman amplifiers are ultimately limited by ASE noise and RIN. Fig. 4 shows the output signal integrated RIN for the five investigated cavity lengths as a function of the FW pump ratio. Please note that the integrated RIN has been normalized to the cavity length $L$ according to the formula below, in order to have a better estimation of the best-performing configuration independently of the required number of spans.

$$
R I N_{d B}^{n o r m}=10 \cdot \log _{10}\left(\frac{1 \mathrm{~km}}{L_{\mathrm{km}}} \int R I N_{\mathrm{lin}}\right)
$$

In the rDFB case with no front-FBG the RIN evolution is essentially flat up to $50 \%$ FPR in all of the cases except for the $10 \mathrm{~km}$ cavity, where the required pump power is substantially higher than in most of the other designs. A higher reflectivity produces a stronger FW propagating Stokes component and, therefore, a greater amount of RIN is transferred from the FW pump to the signal [19]. RIN transfer in short cavities is only marginally affected by an increase of the front reflectivity, whereas cavities longer than $50 \mathrm{~km}$ show a clear inflection point in between $20 \%$ and $30 \%$ FPR. Interestingly, the $150 \mathrm{~km}$ long cavity can tolerate a slightly higher FPR than the $100 \mathrm{~km}$ cavity before the signal RIN starts to increase. We ascribe this behavior to the reduced impact of the BW pump: the BW propagating first Stokes component generated by the BW pump travels a longer length and therefore experiences a higher attenuation. Thus, the portion reflected back in the FW direction by the front FBG is weaker and provides a smaller contribution to the RIN transfer.

Furthermore, in longer cavities, where the interaction length between the FW lasing and the signal is enough for the RIN provided by the pump to be fully transferred to the signal, a clear saturation effect occurs, with the upper bound to the signal RIN being the FW pump RIN. Some of the curves in Fig. 4 couldn't be completed owing to the limited available pump power.

The OSNR measured at the amplifiers output over a $0.1 \mathrm{~nm}$ bandwidth as a function of the FPR is presented in Fig. 5. The partially closed low-reflectivity cavity configurations exhibit somewhat similar performance: as the FW pump contribution increases the output OSNR grows as expected. Longer spans display the larger variation across the FPR range due to the higher attenuation experienced by the signal when the pumping scheme is BW biased, before the amplification from the BW pump takes place. As in the case of the RIN transfer above, the OSNR has been normalized in each case dividing the linear accumulated noise by the total length of the span, in order to compare the relative performance of the amplifier spans with independence of the total transmission length.

The beneficial effect of the FW pumping contribution is barely visible in short cavities up to $50 \mathrm{~km}$ long: the improvement is nearly negligible for FPRs up to 50\% and the overall OSNR enhancement is below $1.5 \mathrm{~dB}$.

On the other hand, the half-open rDFB structure seems to perform rather differently depending on the cavity length: the two 


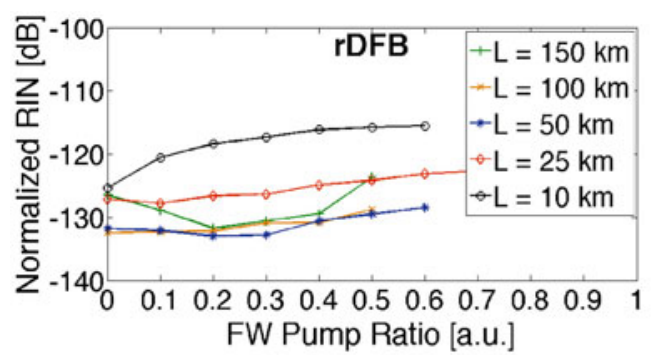

(a)

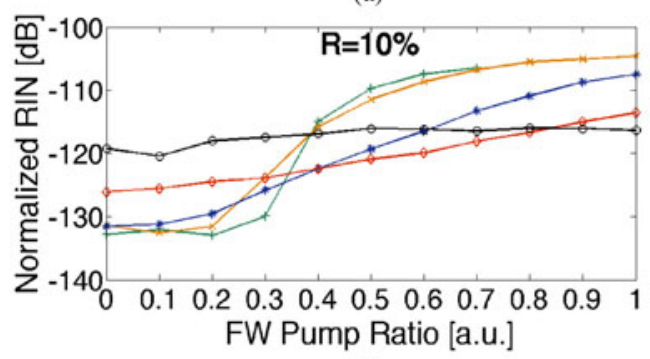

(c)

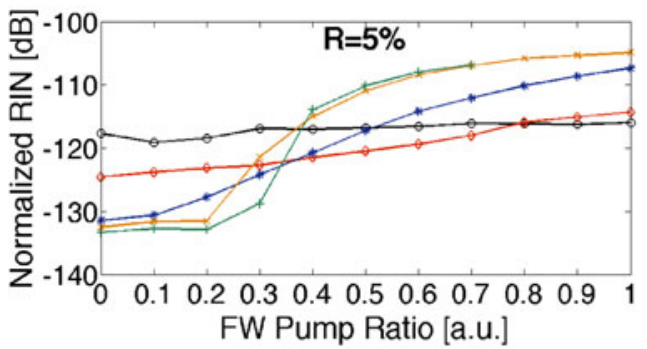

(b)

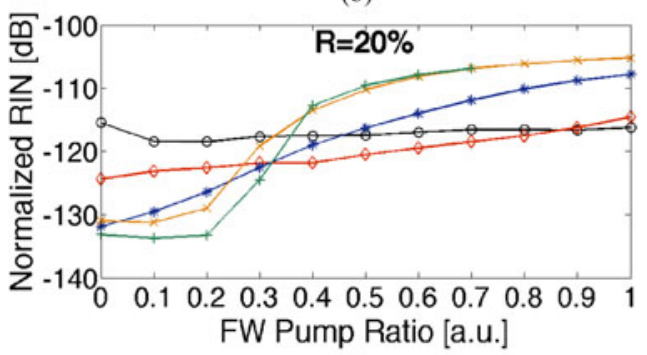

(d)

Fig. 4. Output signal RIN integrated over the [9 kHz, $1 \mathrm{MHz}] \mathrm{RF}$ frequency range for different cavity lengths in the (a) rDFB, (b) $5 \%$, (c) $10 \%$ and (d) $20 \%$ front reflectivity cavity cases. The legend shown in (a) applies to (b), (c) and (d) as well.

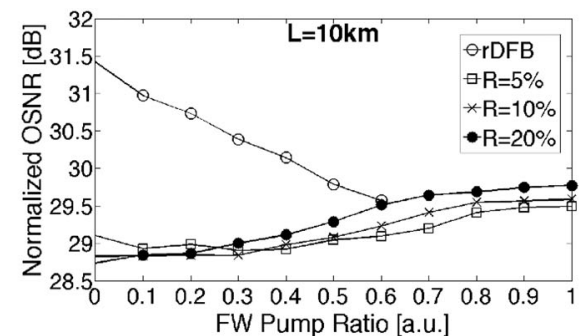

(a)

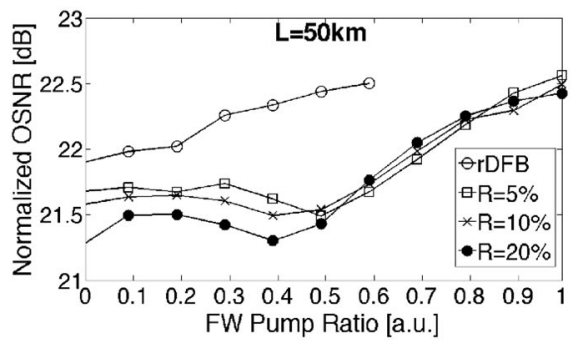

(c)

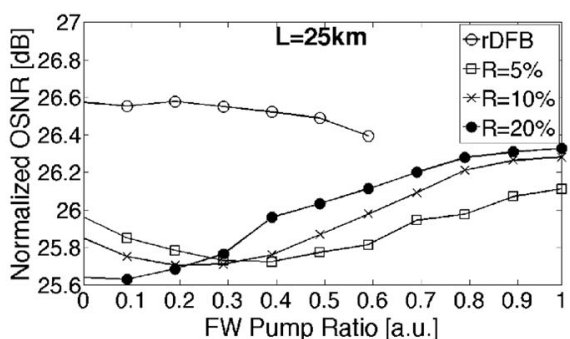

(b)

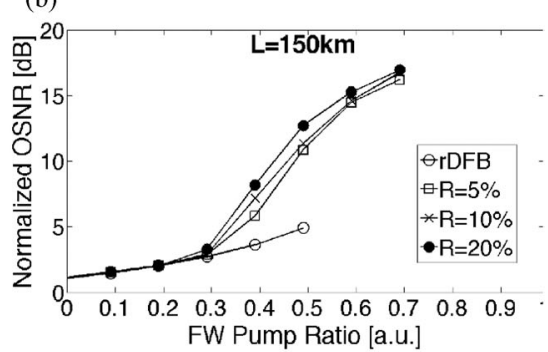

(e)

Fig. 5. Output OSNR for different front-end reflectivities in the (a) $10 \mathrm{~km}$, (b) $25 \mathrm{~km}$, (c) $50 \mathrm{~km}$, (d) $100 \mathrm{~km}$ and (e) $150 \mathrm{~km}$ cavities.

shortest amplifiers perform best when purely counter-pumped as the SMF length is short enough for the BW pump to amplify the signal at the beginning of the span, acting for all practical purposes as a FW pump (see Fig. 6, depicting signal power evolution). An increase in the FW pump contribution only adds noise and therefore reduces the OSNR. For longer lengths, the BW pump reaching the front-end is not strong enough and the signal drops significantly in the first tens of $\mathrm{km}$. As a consequence, FW pumping does have a role in counteracting the fiber loss and the OSNR improves when the FPR increases as shown in Fig. 5(c)-(e). Please note that an unrestricted improvement of the ASE-based OSNR obtained increasing FPR would, as shown in Fig. 4, bring with it a very large increase of the RIN transfer for all spans longer than $50 \mathrm{~km}$. Moreover, after a certain value of FPR, the improvement in OSNR would also be counterbalanced by nonlinearities. Although a close-to symmetrical scheme approaching the lowest power excursion would provide the best balance between noise and nonlinearities [21], and the gained OSNR margin would up to that point translate into a reduced optimal launch power and better overall performance, pushing the FPR beyond this optimal point (typically above $50 \%$ ) would only increase nonlinear penalties. Nevertheless, RIN transfer becomes a major limitation at even lower FPRs of $20 \%$ to $30 \%$, hence being the latter the more restrictive effect 


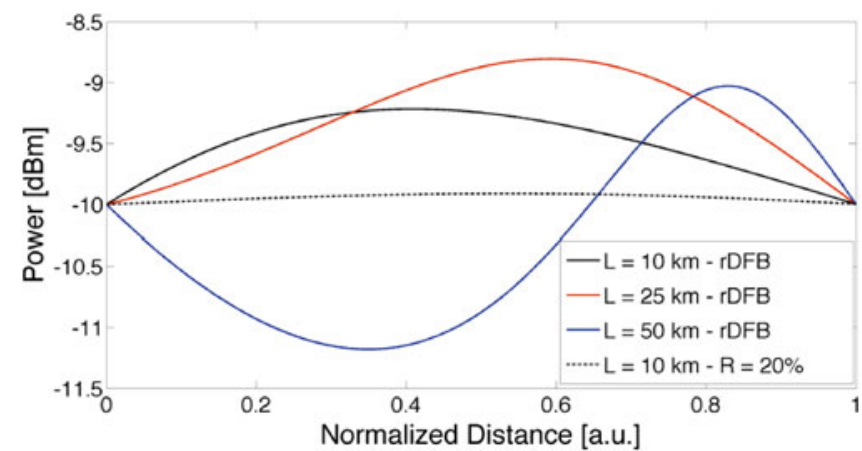

Fig. 6. Simulated signal power evolution for three different cavity lengths in the rDFB configuration with BW-only pumping, and for a $20 \%$ reflectivity, $10 \mathrm{~km}$ cavity (dashed curve).

on forward pump ratio. Generally speaking, the rDFB scheme provides better OSNR than the low-reflectivity closed-cavity URFLs in shorter cells, thanks to a higher contribution of the FW gain (see Fig. 6). On the other hand, in longer cells, where the attenuation experienced by the signal in the initial section of the span is quite high, the favorable effect of a low-reflectivity front-end FBG becomes evident.

Fig. 6 shows the simulated signal power evolution for the three BW pumped rDFB configurations and for the BW pumped, $10 \mathrm{~km}, 20 \%$ reflectivity one.

\section{Pump Depletion Regime}

In order to evaluate the impact of pump depletion in opencavity $\mathrm{rDFB}$ and in URFL, simulations have been performed using the numerical model presented in [13]. The 2nd order transfer function obtained as the ratio of the output signal RIN to the 2nd order FW pump RIN has been calculated for SMF lengths of $10 \mathrm{~km}$ (blue curves), $50 \mathrm{~km}$ (black curves) and $100 \mathrm{~km}$ (red curves) with a FW only pumping. RIN has been calculated as the ratio of the time-averaged mean squared value of the power fluctuations to the squared averaged power of the particular spectral component. The RIN provided by the pump has been considered constant in frequency and equal to $-120 \mathrm{~dB} / \mathrm{Hz}$.

Fig. 7 highlights the effect of pump depletion on the RIN transfer for URFL (a) and rDFB (b) amplifiers. As the signal launch power is increased from $-10 \mathrm{dBm}$ (solid curves) to $0 \mathrm{dBm}$ (dashed curves) and $10 \mathrm{dBm}$ (dash-dot curves) the transfer function remains nearly unchanged for cavity lengths up to $50 \mathrm{~km}$ and no effect of pump depletion can be appreciated in both amplification schemes. For longer cavities, on the other hand, a high signal input power translates into a mitigated RIN transfer in the investigated pump modulation frequency range. Nevertheless, the transfer function in the pump depletion regime does not display a definite $3 \mathrm{~dB}$ corner frequency within the first $100 \mathrm{MHz}$ RF spectrum.

Fig. 8 shows the simulated output OSNR in a $1 \mathrm{~nm}$ bandwidth as a function of the span length for URFL and rDFB architectures with different signal launch powers and 50\% FPR. The effect of pump depletion is negligible for lengths up to $100 \mathrm{~km}$, where the OSNR penalty of the rDFB scheme is around $1 \mathrm{~dB}$ for each of the considered launch powers. Conversely, for longer

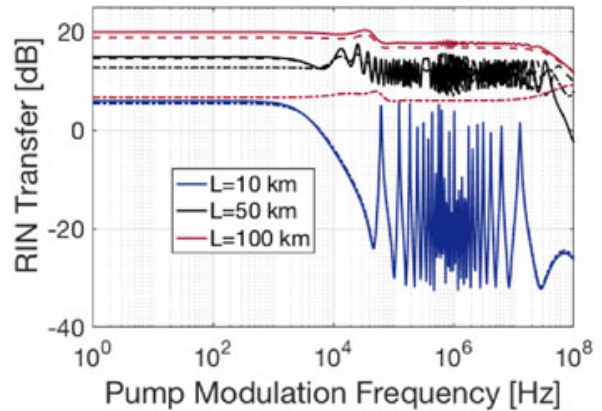

(a)

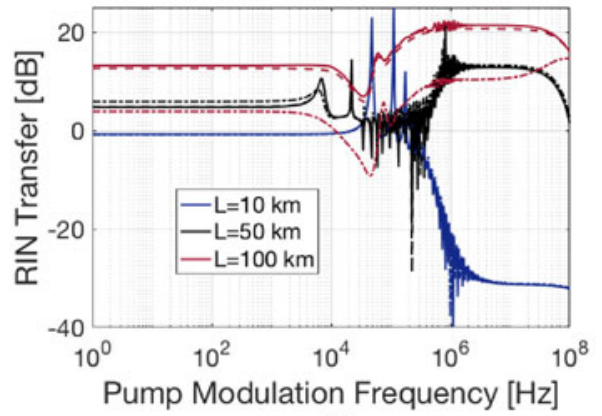

(b)

Fig. 7. Simulated RIN transfer function up to $100 \mathrm{MHz}$ in co-pumped URFL (a) and $\mathrm{rDFB}$ (b) amplification for $10 \mathrm{~km}$ (blue curves), $50 \mathrm{~km}$ (black curves) and $100 \mathrm{~km}$ (red curves) SMF length. The channel launch power is $-10 \mathrm{dBm}$ (solid), $0 \mathrm{dBm}$ (dashed) and $10 \mathrm{dBm}$ (dash-dot).

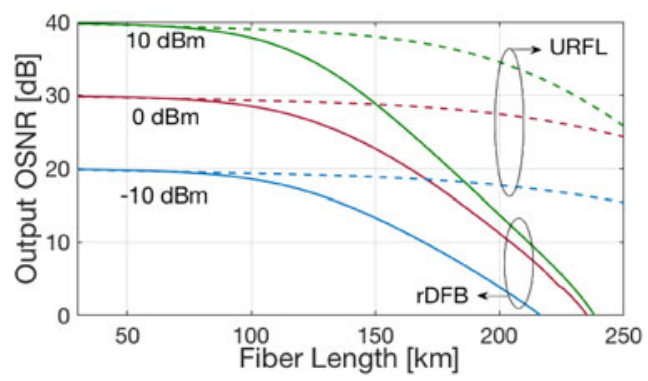

Fig. 8. Simulated output OSNR in a $1 \mathrm{~nm}$ bandwidth for URFL (dashed) and rDFB (solid) configurations with symmetrical bidirectional pumping. Signal input power is $-10 \mathrm{dBm}$ (light blue), $0 \mathrm{dBm}$ (red) and $10 \mathrm{dBm}$ (green).

lengths pump depletion strongly enhances the OSNR difference between the two schemes which, in a $150 \mathrm{~km}$ amplifier, amounts to $5.5 \mathrm{~dB}, 5.9 \mathrm{~dB}$ and $9.2 \mathrm{~dB}$ for $-10 \mathrm{dBm}, 0 \mathrm{dBm}$ and $10 \mathrm{dBm}$ launch power, respectively.

\section{CONCLUSION}

We have performed a thorough experimental study of the performance of a 2nd-order Raman amplifier aimed at the optimization of fundamental design parameters, towards an efficient deployment of distributed amplification in modern transmission systems. Our analysis involves monitoring critical benchmarks such as pump power efficiency, RIN transfer and OSNR as a function of a set of parameters including span length, choice of pumping scheme and front-end reflectivity, scanning the whole 
gamut of operational regimes from pure cavity URFL to rDFB amplification.

Our findings offer insight on the optimal design for cavitybased configurations design, showing the best options to take full advantage of the benefits of Raman amplification without incurring in potentially disruptive effects for the specific applications considered. On the other hand, we find that rDFB architecture can offer advantages in terms of both OSNR and tolerance to RIN transfer only when the cavity length can be chosen to be shorter than $100 \mathrm{~km}$ as it is the case in [7] and [8], and paying a non-negligible price in pump power requirements. The specific case of unrepeatered transmission demands that the cell be as long as possible. Therefore, additional care has to be taken, as the configurations that maximize OSNR require increased front-end reflectivity and strong FW pumping that, in turn, produce the highest RIN transfer from the pumps to the signal. In this scenario, an accurate selection of the design parameters can help improve the performance and efficiency of previously presented unrepeatered systems as in [5], [9] and [10]. Our current results fully support the conclusions on RIN transfer reported in [19], and extend them to other cavity configurations analyzed in this manuscript, showcasing different optimal operational conditions depending on the preferred span length.

\section{REFERENCES}

[1] J. D. Ania-Castañón, "Quasi-lossless transmission using second-order Raman amplification and FBG," Opt. Express, vol. 12, no. 19, pp. 4372-4377, 2004.

[2] J. D. Ania-Castañón, T. J. Ellingham, R. Ibbotson, X. Chen, L. Zhang, and S. K. Turitsyn, "Ultralong raman fiber lasers as virtually lossless optical media," Phys. Rev. Lett., vol. 96, no. 2, 2006, Art. no. 023902.

[3] S. K. Turitsyn et al., "Random distributed feedback fibre laser," Nature Photon., vol. 4, no. 4, pp. 231-235, 2010.

[4] L. E. Nelson, X. Zhou, B. Zhu, M. F. Yan, P. W. Wisk, and P. D. Magill, "All-Raman-amplified, $73 \mathrm{~nm}$ seamless band transmission of $9 \mathrm{~Tb} / \mathrm{s}$ over $6000 \mathrm{~km}$ of fiber," IEEE Photon. Technol. Lett., vol. 26, no. 3, pp. 242-245, Feb. 2014

[5] P. Rosa, J. D. Ania-Castañón, and P. Harper, "Unrepeatered DPSK transmission over $360 \mathrm{~km}$ SMF-28 fibre using URFL based amplification," Opt. Express, vol. 22, no. 8, pp. 9687-9692, 2014.

[6] M. Tan, P. Rosa, S. Thai Le, I. D. Phillips, and P. Harper, "Evaluation of $100 \mathrm{G}$ DP-QPSK long-haul transmission performance using second order co-pumped Raman laser based amplification," Opt. Express, vol. 23, no. 17 , pp. 22181-22189, 2015
[7] M. Tan, P. Rosa, S. T. Le, Md. A. Iqbal, I. D. Phillips, and P. Harper, "Transmission performance improvement using random DFB laser based Raman amplification and bidirectional second-order pumping," Opt. Express, vol. 24, no. 3, pp. 2215-2221, 2016.

[8] M. Tan, P. Rosa, I. Phillips, and P. Harper, "Extended reach of $116 \mathrm{~Gb} / \mathrm{s}$ DP-QPSK transmission using random DFB fiber laser based Raman amplification and bidirectional second-order pumping," in Proc. Opt. Fiber Commun. Conf., Los Angeles, CA, USA, 2015, pp. 1-3.

[9] L. Galdino et al., "Unrepeatered nyquist PDM-16 QAM transmission over $364 \mathrm{~km}$ using Raman amplification and multi-channel digital backpropagation," Opt. Lett., vol. 40, no. 13, pp. 3025-3028, 2015.

[10] P. Rosa et al., "Unrepeatered DP-QPSK transmission over $352.8 \mathrm{~km} \mathrm{SMF}$ using random DFB fiber laser amplification," IEEE Photon. Technol. Lett., vol. 27, no. 11, pp. 1189-1192, Jun. 2015.

[11] C. R. S. Fludger, V. Handerek, and R. J. Mears, "Pump to signal RIN transfer in Raman fiber amplifiers," J. Lightw. Technol., vol. 19, no. 8, pp. 1140-1148, Aug. 2001.

[12] M. Krause, S. Cierullies, H. Renner, and E. Brinkmeyer, "Pump-to-Stokes RIN transfer in Raman fiber lasers and its impact on the performance of copumped Raman amplifiers," Opt. Commun., vol. 260, no. 2, pp. 656-661, 2006

[13] M. Alcón-Camas and J. D. Ania-Castañón, "RIN transfer in 2nd-order distributed amplification with ultralong fiber lasers," Opt. Express, vol. 18, no. 23, pp. 23569-23575, 2010.

[14] J. Nuño, M. Alcon-Camas, and J. D. Ania-Castañón, "RIN transfer in random distributed feedback fiber lasers, "Opt. Express, vol. 20, no. 24, pp. 27376-27381, 2012.

[15] I. Phillips et al., "Exceeding the nonlinear-Shannon limit using Raman laser based amplification and optical phase conjugation," in Proc. Opt. Fiber Commun. Conf., San Francisco, CA, USA, 2014, pp. 1-3.

[16] L. Galdino et al., "Amplification schemes and multi-channel DBP for unrepeatered transmission," J. Lightw. Technol., vol. 34, no. 9, pp. 22212227, May 2016

[17] P. Rosa, S. Thai Le, G. Rizzelli, M. Tan, and J. D. Ania-Castañón, "Signal power asymmetry optimisation for optical phase conjugation using Raman amplification," Opt. Express, vol. 23, no. 25, pp. 31772-31778, 2015.

[18] M. Tan et al., "RIN mitigation in second-order pumped Raman fibre laser based amplification," in Proc. Asia Commun. Photon. Conf., OSA Tech. Dig., 2015, Paper AM2E.6.

[19] G. Rizzelli et al., "Impact of input FBG reflectivity and forward pump power on RIN transfer in ultralong Raman laser amplifiers," Opt. Express, vol. 24, no. 25, pp. 29170-29175, 2016.

[20] J. D. Ania-Castañón, V. Karalekas, P. Harper, and S. K. Turitsyn, "Simultaneous spatial and spectral transparency in ultralong fiber lasers," Phys. Rev. Lett., vol. 101, no. 12, 2008, Art. no. 123903.

[21] J. C. Bouteiller, K. Brar, and C. Headley, "Quasi-constant signal power transmission," in Proc. 28th Eur. Conf. Opt. Commun., Copenhagen, Denmark, 2002, pp. 1-2.

Authors' biographies not available at the time of publication. 